\title{
Development of Algorithm and System for Automatic Generation of Nursing Summaries from Nursing Care Plans
}

\author{
Misao Miyagawa', Yuko Yasuhara², Tetsuya Tanioka², Hirokazu Ito², Motoyuki Suzuki ${ }^{3}$, \\ Rozzano Locsin 4 \\ ${ }^{1}$ Department of Nursing, Faculty of Health and Welfare, Tokushima Bunri University, Tokushima, Japan \\ ${ }^{2}$ Institute of Health Biosciences, The University of Tokushima Graduate School, Tokushima, Japan \\ ${ }^{3}$ Faculty of Information Science and technology, Osaka Institute of Technology, Osaka, Japan \\ ${ }^{4}$ Christine E. Lynn College of Nursing, Florida Atlantic University, Boca Raton, USA \\ Email: miyagawa@tks.bunri-u.ac.jp
}

Received 27 February 2014; revised 26 March 2014; accepted 25 April 2014

Copyright (C) 2014 by authors and Scientific Research Publishing Inc.

This work is licensed under the Creative Commons Attribution International License (CC BY).

http://creativecommons.org/licenses/by/4.0/

(c) (i) Open Access

\begin{abstract}
A nursing care planning system that automatically generated nursing summaries from information entered into the Psychiatric Outcome Management System (PSYCHOMS $®$, Tanioka et al.), was developed to enrich the content of nursing summaries at psychiatric hospitals, thereby reducing the workload of nurses. Preparing nursing summaries entails finding the required information in nursing records that span a long period of time and then concisely summarizing this information. This time consuming process depends on the clinical experience and writing ability of the nurse. The system described here automatically generates the text data needed for nursing summaries using an algorithm that synthesizes patient information recorded in electronic charts, the Nursing Care Plan information or the data entered for North American Nursing Diagnosis Association (NANDA) 13 domains with predetermined fixed phrases. Advantages of this system are that it enables nursing summaries to be generated automatically in real time, simplifies the process, and permits the standardization of useful nursing summaries that reflect the course of the nursing care provided and its evaluation. Use of this system to automatically generate nursing summaries will allow more nursing time to be devoted to patient care. The system is also useful because it enables nursing summaries that contain the required information to be generated regardless of who prepares them.
\end{abstract}

\section{Keywords}

Nursing Summary Document, Nursing Care Plan, Automatic Generation, PSYCHOMS

How to cite this paper: Miyagawa, M., Yasuhara, Y., Tanioka, T., Ito, H., Suzuki, M. and Locsin, R. (2014) Development of Algorithm and System for Automatic Generation of Nursing Summaries from Nursing Care Plans. Intelligent Information Management, 6, 97-103. http://dx.doi.org/10.4236/iim.2014.63011 


\section{Introduction}

A nursing summary provides a synopsis of the course of the patient's hospitalization for a certain period of time and the received nursing care [1]. This is needed if there is a change in the patient's condition which can be communicated to other health care team members at the next facility. In doing so, nursing care can be provided with continuity and without delay [2]. In addition, nursing summaries are prepared periodically so that the care of patients' requiring long-term hospitalization is reviewed and the nursing care that has been provided is reexamined in order to provide more appropriate nursing.

Many patients' requiring long-term care hospitalizations are in psychiatric long-term-care beds and in longterm-care beds for the elderly if there is no change in the patient's condition, the same information must be recorded repeatedly in the nursing summary. This is inefficient and costly for the nursing service. In preparing a nursing summary, the nurse must enter or record the required information on the designated form from the beginning, and at discharge, they must find the required events in a nursing record that spans a long period of time and then concisely summarize the information. Consequently, preparation of the nursing summary depends on the extent to which the nurse understands the patient's condition, the nurse's clinical capabilities, and their writing ability [3]. Because that preparing the summary requires time and effort, it is a burden on the nurse [4] [5]. Moreover, it has been noted that the information recorded is sometimes inadequate [6].

Previous studies have examined the methods and systems used in preparing summaries [7] [8]. Studies have also been conducted on electronic summary preparations in the healthcare setting [9] [10]. For example, there are systems that extract text that includes specific expressions from the text portion of the information in electronic charts, delete text such as conjunctions and dates from the extracted text, and output a summary [11]. Other systems have the extraction and classification functions by a disease name from an electronic medical record. Also this system can create the summary according to the classified disease name [12]. With these summary preparation systems, however, it becomes apparently difficult to find the necessary information as the amount of information recorded in the electronic chart increases, and when summaries are prepared at regular intervals, the information needed treatment or nursing is extracted manually.

The nursing summary preparation systems that have been introduced in the clinical setting in Japan automatically extract basic patient information such as the diagnosis from the electronic chart [13]-[16]. This to some extent reduces the work involved in manually entering text to prepare the summary.

The nursing summary is a summary of the nursing process itself rather than simply a list of data, and it must be edited into data with a high level of added value that includes the considerations required for nursing care continuity [17] [18]. However, a system that automatically generates these types of useful nursing summaries has not been developed.

A nursing care plan is very important record in the nurse's record in the electronic chart. Included in this record are the nursing problems extracted by the assessment of the patient's condition, a nursing care plan for addressing these problems, the implementation status of the plan, and an evaluation of it. These problems could be solved if the nursing care plan information and the plan evaluation, which are entered by the nurse, could be synthesized with predetermined fixed phrases to automatically generate up-to-date text data.

The aim of this study was to construct a system that automatically generates nursing summaries using data entered into the PSYCHOMS ${ }^{\circledR}$ nursing care planning system.

\section{Methods}

\subsection{Nursing Care Plan and Nursing Summary}

The nursing summary explains the effectiveness of the nursing care provided to the patient and the extent to which the expected results have been achieved. It clarifies what nursing care should be continued in the future and is needed to ensure the evaluation and continuity of nursing care. The information in the summary includes patient attributes, information on drugs and other treatments to be continued, hospitalization status and nursing course, and nursing diagnoses and nursing care details requiring continuity [19] [20].

The nurse ascertains the nursing-related problems of the individual patient and takes responsibility for independently implementing the nursing intervention needed in the process of treatment and nursing care [20]. To accomplish this, the nurse performs a nursing diagnosis, establishes and implements a nursing care plan (nursing objectives and intervention plan) for each diagnosis established, evaluates the efficacy of the plan at regular in- 
tervals, and revises it as necessary. Consequently, the information in the nursing care plan includes information needed for the nursing summary. In other words, the objectives of the nursing summary can be satisfied by accurately extracting and summarizing the information recorded in the nursing care planning system. However, the nurse must find a tacit algorithm to formulate a procedure for extracting the information needed to reexamine the continuous nursing care they conceive of and the provided nursing care.

\subsection{Automatic Generation of Nursing Summaries from Nursing Care Plans}

\subsubsection{Overview of System for Automatically Generating Nursing Summaries}

The overview of this system is shown in Figure 1. Because the information recorded in the nursing summary basically is determined according to the situation (e.g., at admission, when transferring to another hospital), it can be sufficiently expressed with fixed phrases that include the necessary information.

The method used to generate the text of the summary in this system involves preparing a template of fixed phrases for each situation (admission, mid-hospitalization, ward transfer, discharge, and hospital transfer) in advance, extracting the required information from the nursing care plan data, and then inserting it into the appropriate template.

This system uses nursing care plan data in multiple data formats that are commonly used at present, rather than a single format. Because the types of recorded information vary, a fixed phrase template is needed for each data format.

Moreover, because the information included in the nursing care plan data varies, the summaries are output also vary. Therefore, when generating a summary, the user selects the nursing care plan data they require (and the corresponding fixed phrase template).

When the user specifies the patient name, the System Controller $1\left(\mathrm{SC}_{1}\right)$ shown in the Figure 1, judge whether a nursing summary is created from the Nursing Care Plan or the North American Nursing Diagnosis Association (NANDA) 13 domains. Next, data required for a nursing summary is acquired from the PSYCHOMS database. The System Controller $2\left(\mathrm{SC}_{2}\right)$ extracts the required information from the nursing care plan data for that patient and incorporates it into the corresponding fixed phrase template to automatically generate the nursing summary.

\subsubsection{Phrase Templates}

Fixed phrase templates were prepared for each nursing care plan data format and situation in order to generate summaries. The system accommodates two nursing care plan data formats: a format for patient information obtained from the PSYCHOMS ${ }^{\circledR}[21]$ and a format for the Nursing Care Plan information or the NANDA 13 domains.

Moreover, because that summaries can be generated for five situations: admission, mid-hospitalization, ward transfer, discharge, and hospital transfer, a total of 10 types of fixed phrase templates can be prepared manually.

The Nursing Care Planning System of the PSYCHOMS ${ }^{\circledR}$ can be used in two ways: 1 ) to create a nursing care plan from the nursing diagnosis, and 2) to create a nursing care plan from the NANDA 13 domains.

The template used to create a nursing care plan from the nursing diagnosis (patient information obtained from PSYCHOMS ${ }^{\circledR}$ ) is shown in Figure 2.

As the example indicates, the templates consist of sentences interspersed with blank spaces, and words and phrases extracted from the nursing care plan data are inserted into the spaces to generate the nursing summary. The nursing care plan data are not simply sentences but rather the information is structured and classified according to attribute (e.g., nursing diagnosis, assessment). Consequently, in extracting information, the information for the specified attributes can be used unchanged.

An example of a fixed phrase template for the NANDA13 domains is shown in Figure 3. With this nursing care plan data format, a nursing diagnosis is entered after being extracted from the results of an assessment of the patient's physical, psychological, and social condition based on the NANDA 13 domains. Consequently, the NANDA domains and specific patient conditions and circumstances that are problematic are documented.

\section{Results}

This system is a Japanese system, therefore the output examples of summary text automatically generated from the nursing care plan are shown in Figure 4 and Figure 5 in Japanese language. In the Figure 4, it shows auto- 


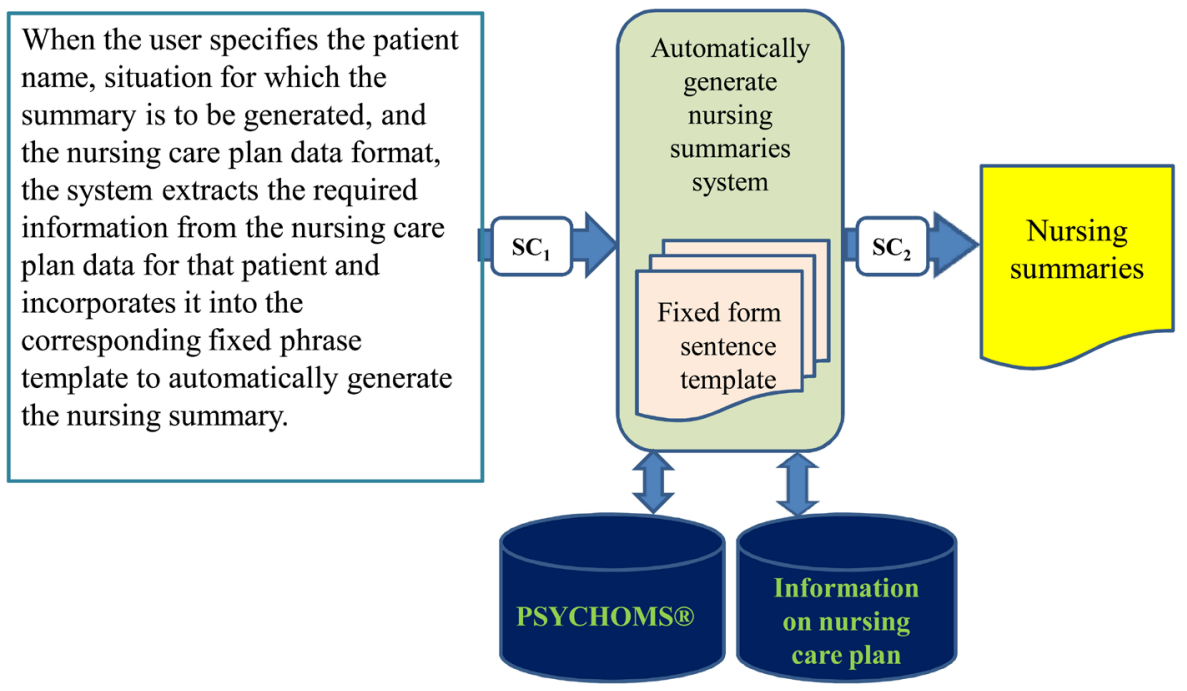

Figure 1. Automatic summary generation algorithm.

The currently proposed nursing diagnosis is \# (Problem No.) (Nursing diagnosis). Support was provided for (Assessment), the problem associated with \# (Problem No.) (Nursing diagnosis), with (Observation parameter) as the observation parameter, (Support parameter) as the support parameter, and (Education parameter) as the education parameter. Currently, the condition is (Latest evaluation).

Figure 2. Format A, for summary text based on the Nursing Care Plan.

The currently proposed nursing diagnosis based on the 13 NANDA domains is (Nursing diagnosis) of domain (Domain No.) (Domain name), and the symptoms/condition are (Symptoms/Condition) (Specific condition/Circumstances).

Figure 3. Format B, for summary text based on the NANDA 13 domains.

matically generated result from the Nursing Care Plan. The generated text reflected nursing intervention implemented to address the nursing diagnosis and the evaluation performed after the intervention result appropriately. In the Figure 5, it shows automatically generated result from the NANDA 13 domains. Also, the generated text reflected the nursing diagnosis, patient's psychiatric symptoms and conditions appropriately.

\section{Discussion}

The nursing summary must elucidate the effectiveness of the nursing care provided to the patient and the extent to which the expected results have been achieved and clarify what nursing care should be continued in the future. Also, in order to provide nursing with continuity, the up-to-date nursing information required must be accurately reflected in the nursing summary. In this respect, the generated summary text by this system provided the information required for the nursing summary [22]-[25].

The system synthesizes the nursing care plan information and its evaluation, which is entered by the nurse, with predetermined fixed phrases to automatically generate up-to-date text data. Therefore, it has the advantage of ensuring that the information in the automatically generated nursing summaries is accurate and timely.

Furthermore, when generating a nursing summary, nursing diagnoses that were resolved and finalized through the nursing intervention also could be included in the nursing summary as part of the nursing course. In other 


\begin{tabular}{|l|}
\hline 要約(転院時の看護上の問題点) \\
現在、看護診断として立案しているのは\#2自己同一性混乱である。 \\
\#2「自己同一性混乱」の問題である奇妙な行動、退行行動、自我境界 \\
の損失、不安に対して観察項目として「表」、退行行動の状況」、援助項 \\
目として安心できる環境を提供する」、教育項目として患者が現実を受け \\
入れ、現実とのかかわりを続けることに役立つような活動を指導する」援助を \\
行った。現在は「奇妙な行動が少なくなったが、不安の訴えが続いている」と \\
いら状況である。
\end{tabular}

Figure 4. The generated summary text: automatically generated from the Nursing Care Plan.

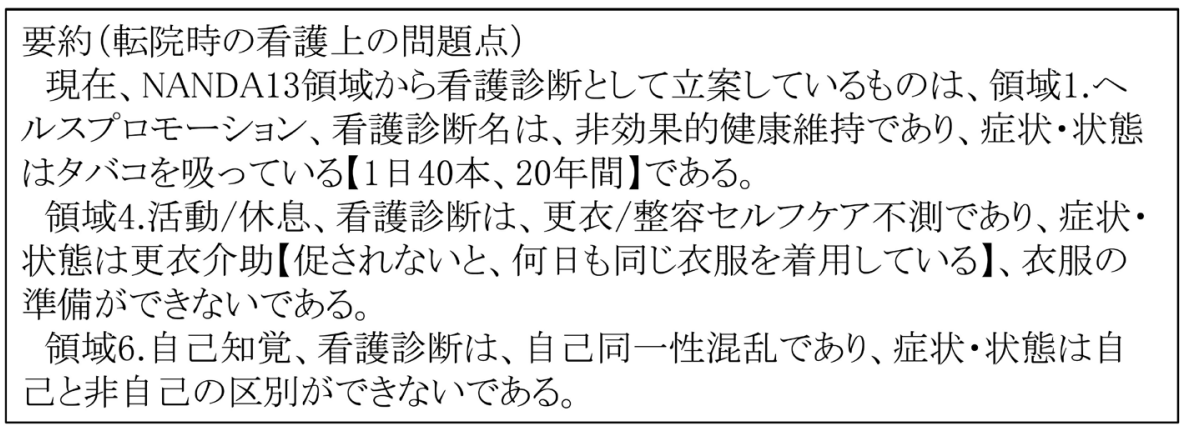

Figure 5. The generated summary text: automatically generated from the NANDA 13 domains.

words, the nursing summary includes a description of the change in the individual subject's condition and indicates the problems predicted after discharge, which is the purpose of the summary, thereby ensuring nursing continuity and enabling the nursing to be evaluated. The nursing summaries generated by this system include sufficient up-to-date information for nursing continuity and therefore benefit both the patient and the nurse.

Personnel shortages are a serious problem for psychiatric hospitals and long-term-care beds. However, the use of this system would eliminate the need of repeated preparing summaries that contain the same information for patients in the chronic phase, who have few changes in their condition. Therefore, the time saved by generating the summaries automatically could be effectively utilized to provide the nursing care needed.

In this system, because that a nursing diagnosis and plan can be proposed for the acute phase based on the NANDA 13 domains, the system can be applied to generating nursing summaries for other fields in addition to psychiatry. The NANDA nursing diagnoses used by this system represent a universal language [21] [26], which means that the automatic summary generation system created based on this study has broad utility not only in Japan but also in other countries.

As human factors, the appropriateness of the nursing summary depends on the ability of the nurse using the system. Generated nursing summary based on the nursing care plan and evaluation entered by the nurse, this information must be properly entered. It will be necessary in the future to increase the accuracy of the system to improve the readability of the automatically generated summaries and to provide training for nursing personnel to ensure that the nursing summaries are of high quality. Proper entry of the nursing care plan and evaluation are important for ensuring that the nursing summary generated is of high quality. Moreover, if there are many persistent nursing problems, the size of the summary increases, and it becomes difficult to read. It therefore became apparent that a feature was needed for revising the text appropriately using a feature that enables a human nurse to edit the summary extracted by the automatic summary feature. Based on the study results and clinical evaluation, the addition of these features has made the current system easier to use in the clinical setting.

\section{Conclusions}

This study focuses on nursing care plan data, and proposes a method of automatically generating nursing summaries from the information in nursing care plans. This system was developed to synthesize the nursing diagno- 
sis and evaluation entered by the nurse nursing care plan and the NANDA 13 domains in the PSYCHOMS ${ }^{\circledR}$ with predetermined fixed phrases and automatically generated up-to-date text data.

For psychiatric hospitals, which have many patients who require long-term hospitalization or are admitted and discharged repeatedly, the system enables nursing summaries that include useful patient information to be generated automatically and the process of summary generation to be simplified and more timely.

\section{Acknowledgements}

This study was supported by a grant for Strategic Information and Communications R\&D Promotion Program (SCOPE) of Japan (No. 122309008).

\section{References}

[1] Hübner, U., Flemming, D., Heitmann, K.U., Oemig, F., Thun, S. and Dickerson, A. (2010) The Need for Standardized Documents in Continuity of Care: Results of Standardising the eNursing Summary. Studies in Health Technology and Informatics, 160, 1169-1173.

[2] Tjia, J., Bonner, A., Briesacher, B.A., McGee, S., Terrill, E. and Miller, K. (2009) Medication Discrepancies upon Hospital to Skilled Nursing Facility Transitions. Journal of General Internal Medicine, 24, 630-635. http://dx.doi.org/10.1007/s11606-009-0948-2

[3] Kuusisto, A., Asikainen, P., Lukka, H. and Tanttu, K. (2009) Experiences with the Electronic Nursing Discharge Summary. Studies in Health Technology and Informatics, 146, 226-230.

[4] Needleman, J., Kurtzman, E.T. and Kizer, K.W. (2007) Performance Measurement of Nursing Care: State of the Science and the Current Consensus. Medical Care Research and Review, 64, 10S-43S. http://dx.doi.org/10.1177/1077558707299260

[5] Lomas, C. (2012) The Burden of Bureaucracy. Nursing Standard, 26, 22-24. http://dx.doi.org/10.7748/ns2012.03.26.30.22.p7892

[6] Walz, S.E., Smith, M., Cox, E., Sattin, J. and Kind, A.J. (2011) Pending Laboratory Tests and the Hospital Discharge Summary in Patients Discharged to Sub-Acute Care. Journal of General Internal Medicine, 26, 393-398. http://dx.doi.org/10.1007/s11606-010-1583-7

[7] Ferreira, R., Freitas, F., Cabral, L.S., Lins, R.D., Lima, R. and Franca, G. (2013) A Four Dimension Graph Model for Automatic Text Summarization. Proceedings of International Joint Conferences on Web Intelligence (WI) and Intelligent Agent Technologies (IAT), Atlanta, 17-20 November 2013, 389-396.

[8] Hammond, K., Efthimiadis, E., Laundry, R., Thielke, S., Weir, C. and Embi, P. (2011) Preliminary Validation of a Method to Measure Information Value in Clinical Documentation. Proceedings of 44th Hawaii International Conference on System Sciences (HICSS), Kauai, Hawaii USA, 1- 7.

[9] Quan, S. and Tsai, O. (2007) Signing on to Sign out, Part 2: Describing the Success of a Web-Based Patient Sign-Out Application and How It Will Serve as a Platform for an Electronic Discharge Summary Program. Healthcare Quarterly, 10, $120-124$.

[10] Kawanaka, H., Shiroyama, Y., Tsuruoka, S., Shinogi, T. and Yamamoto, K. (2008) A Study on Document Structure Recognition of Discharge Summaries for Analogous Case Search System. Proceedings of the 8th IAPR International Workshop on Document Analysis Systems, Nara, 16-19 September 2008, 423-430.

[11] Kawajiri, H. (2005) The Summary Generation Method and Its Program. Japan Patent No. P2005-174003A, in Japanese.

[12] Yamane, Y. (2009) A Summary Creation Device, a Summary Creation System, and Its Summary Creation Program. Japan Patent No. JP2009-157539A, in Japanese.

[13] Gene Com (2012) A Cooperation Solution with an Electronic Health Record System. In Japanese. http://www.genecom.co.jp/record/iryou/entry-355.html

[14] Care Com (2013) NiCSS-R8, Nursing Support System. In Japanese. http://www.carecom.jp/product/medical/nursecall/item01/r8/nic_option.html

[15] Toshiba Information Systems (Japan) Corporation (2014) I-MEDIC, Nursing Support System. http://www.tjsys.co.jp/page.jsp?id=2795

[16] Hübner, U., Saranto, K., Coenen, A. and Sensmeier, J. (2013) eNursing Summary-Where Global Standardisation and Regional Practice Meet. Medinfo 2013. http://person.hst.aau.dk/ska/MEDINFO2013/AllPresentations/Pdfs/Panels/A11_961_MEDINFO2013.pdf

[17] Ferraro-McDuffie, A. and Booker, K.D. (1993) Documenting the Primary Nursing Summary Note: A Leap toward 
Professionalism. Pediatric Nursing, 19, 189-193.

[18] Lagoe, R.J. and Westert, G.P. (2006) Community Wide Electronic Distribution of Summary Health Care Utilization Data. BMC Medical Informatics and Decision Making, 6, 17.

[19] Olsen, R.M., Hellzen, O. and Enmarker, I. (2013) Nurses’ Information Exchange during Older Patient Transfer: Prevalence and Associations with Patient and Transfer Characteristics. International Journal of Integrated Care, 13, e005.

[20] Snyder, M., Egan, E.C. and Nojima, Y. (1996) Defining Nursing Interventions. Image: The Journal of Nursing Scholarship, 28, 137-141. http://dx.doi.org/10.1111/j.1547-5069.1996.tb01206.x

[21] Tanioka, T., Kawamura, A., Date, M., Osaka, K., Yasuhara, Y. and Kataoka, M. (2010) Computerized Electronic Nursing Staffs' Daily Records System in the “A” Psychiatric Hospital. Present Situation and Future Prospects. Proceedings of 2010 International Conference on Natural Language Processing and Knowledge Engineering, Beijing, 21-23 August 2010, 1-4.

[22] Lyons, K.J., Giordano, C., Speakman, E., Isenberg, G., Antony, R. and Hanson-Zalot, M. (2013) Jefferson Interprofessional Clinical Rounding Project: An Innovative Approach to Patient Care. Journal of Allied Health, 42, 197-201.

[23] Westra, B.L., Subramanian, A., Hart, C.M., Matney, S.A., Wilson, P.S. and Huff, S.M. (2010) Achieving "Meaningful Use” of Electronic Health Records through the Integration of the Nursing Management Minimum Data Set. Journal of Nursing Administration, 40, 336-343. http://dx.doi.org/10.1097/NNA.0b013e3181e93994

[24] Blake-Mowatt, C., Lindo, J.L.M. and Bennett, J. (2013) Evaluation of Registered Nurses’ Knowledge and Practice of Documentation at a Jamaican Hospital. International Nursing Review, 60, 328-334. http://dx.doi.org/10.1111/inr.12040

[25] Welton, J.M. and Halloran, E.J. (2005) Nursing Diagnoses, Diagnosis-Related Group, and Hospital Outcomes. Journal of Nursing Administration, 35, 541-549. http://dx.doi.org/10.1097/00005110-200512000-00008

[26] Rutherford, M. (2008) Standardized Nursing Language: What Does It Mean for Nursing Practice? OJIN: The Online Journal of Issues in Nursing, 13. http://www.nursingworld.org/ojin/ 